\title{
Regional Fluid-Attenuated Inversion Recovery (FLAIR) at 7 Tesla correlates with amyloid beta in hippocampus and brainstem of cognitively normal elderly subjects
}

\author{
Simon J. Schreiner ${ }^{1+}$, Xinyang Liu ${ }^{2+}$, Anton F. Gietl ${ }^{1}$, Michael Wyss ${ }^{3}$, Stefanie C. Steininger ${ }^{1}$, \\ Esmeralda Gruber ${ }^{1}$, Valerie Treyer ${ }^{1,4}$, Irene B. Meier ${ }^{1,5}$, Andrea M. Kälin ${ }^{1}$, Sandra E. Leh ${ }^{1}$, Alfred Buck ${ }^{4}$, \\ Roger M. Nitsch ${ }^{1}$, Klaas P. Pruessmann ${ }^{3}$, Christoph Hock ${ }^{1}$ and Paul G. Unschuld ${ }^{1 *}$ \\ ' Division of Psychiatry Research and Psychogeriatric Medicine, University of Zürich, Zürich, Switzerland \\ ${ }^{2}$ Department of Radiology, Harvard Medical School, Brigham and Women's Hospital, Boston, MA, USA \\ ${ }^{3}$ Department of Information Technology and Electrical Engineering, Institute for Biomedical Engineering, University of Zürich and ETH Zürich, Zürich, Switzerland \\ ${ }^{4}$ Division of Nuclear Medicine, University of Zürich, Zürich, Switzerland \\ ${ }_{5}^{5}$ Taub Institute for Research on Alzheimer's Disease and the Aging Brain, College of Physicians and Surgeons, Columbia University Medical Center, \\ New York, NY, USA
}

Edited by:

Manuel Menéndez-González, Hospital Álvarez-Buylla, Spain

Reviewed by:

Federica Agosta, Vita-Salute San

Raffaele University, Italy

Kenichi Oishi, Johns Hopkins

University, USA

\section{*Correspondence}

Paul G. Unschuld, Division of

Psychiatry Research and

Psychogeriatric Medicine, University

of Zürich, Minervastrasse 145,

$\mathrm{CH}-8032$ Zürich, Switzerland

e-mail: paul.unschuld@uzh.ch

these authors have contributed equally to this work.
Background: Accumulation of amyloid beta $(A \beta)$ may occur during healthy aging and is a risk factor for Alzheimer Disease (AD). While individual $A \beta$-accumulation can be measured non-invasively using Pittsburgh Compund-B positron emission tomography (PiB-PET), Fluid-attenuated inversion recovery (FLAIR) is a Magnetic Resonance Imaging (MRI) sequence, capable of indicating heterogeneous age-related brain pathologies associated with tissue-edema. In the current study cognitively normal elderly subjects were investigated for regional correlation of PiB- and FLAIR intensity.

Methods: Fourteen healthy elderly subjects without known history of cognitive impairment received 11C-PiB-PET for estimation of regional A $\beta$-load. In addition, whole brain T1-MPRAGE and FLAIR-MRI sequences were acquired at high field strength of 7 Tesla (7T). Volume-normalized intensities of brain regions were assessed by applying an automated subcortical segmentation algorithm for spatial definition of brain structures. Statistical dependence between FLAIR- and PiB-PET intensities was tested using Spearman's rank correlation coefficient (rho), followed by Holm-Bonferroni correction for multiple testing.

Results: Neuropsychological testing revealed normal cognitive performance levels in all participants. Mean regional PiB-PET and FLAIR intensities were normally distributed and independent. Significant correlation between volume-normalized PiB-PET signals and FLAIR intensities resulted for Hippocampus (right: rho $=0.86$; left: rho $=0.84$ ), Brainstem (rho $=0.85$ ) and left Basal Ganglia vessel region ( $r$ ho $=0.82$ ).

Conclusions: Our finding of a significant relationship between PiB- and FLAIR intensity mainly observable in the Hippocampus and Brainstem, indicates regional $A \beta$ associated tissue-edema in cognitively normal elderly subjects. Further studies including clinical populations are necessary to clarify the relevance of our findings for estimating individual risk for age-related neurodegenerative processes such as AD.

Keywords: PiB-PET, MRI, 7 Tesla, amyloid beta, FLAIR, aging

\section{INTRODUCTION}

Aging of the human brain is associated with increased accumulation of extracellular Amyloid beta (A $\beta$ ) (Rodrigue et al., 2012), which can be non-invasively measured by positron emission tomography using radioactively labeled stains such as 11-C Pittsburgh Compund-B (PiB-PET) (Klunk et al., 2004; Vandenberghe et al., 2010). While spreading of $A \beta$-deposits is a risk factor for age-related cognitive decline and a pathological hallmark of Alzheimer Disease (AD) (Alzheimer, 1907; Hock and
Nitsch, 2000; Jack et al., 2009; Sperling et al., 2011), A $\beta$ related brain change may take place decades before manifestation of $\mathrm{AD}$ as reflected by neuronal dysfunction, region-specific brain atrophy or subtle neuropsychological deficits (Mormino et al., 2009; Sheline et al., 2010; Sperling et al., 2013; Steininger et al., 2014). However, as data from postmortem neuropathological assessment show that a considerable share of elderly individuals with brain $\mathrm{A} \beta$ - deposition never experienced AD (Price and Morris, 1999; Knopman et al., 2003; Savva et al., 2009), investigation of 
$\mathrm{A} \beta$-associated effects on brain tissue of non-demented individuals remains a research question of particular interest (Riudavets et al., 2007; Iacono et al., 2008; Steffener and Stern, 2012).

Fluid-attenuated inversion recovery (FLAIR) is a magnetic resonance imaging (MRI) contrast based on tissue T2 prolongation without cerebrospinal fluid (CSF) signal interference (De Coene et al., 1992). While FLAIR-based contrasts are routinely used in cerebral MRI for imaging of tissue-edema, regional FLAIR hyperintensities have been shown to relate to progression of many brain diseases but also to reflect a wide variety of pathological conditions associated with aging (Young et al., 2008; Neema et al., 2009). FLAIR MRI significantly benefits from high magnetic field strength, as shown by increased signal to noise ratio (SNR) when performing FLAIR at 7 Tesla vs. 3 Tesla or 1.5 Tesla, respectively (Visser et al., 2010; Zwanenburg et al., 2010).

Based on these earlier reports, we hypothesized that potential $\mathrm{A} \beta$ - associated alterations in the aging brain may be indicated by local tissue-edema as reflected by increased FLAIR signal before manifestation of neurocognitive impairment and moreover take place in brain regions with particular relevance for age-related neurodegenerative pathology.

To answer this question, cognitively normal elderly subjects were administered PiB-PET for measuring brain A $\beta$-load and also MRI for quantitative assessment of regional FLAIR intensities. FLAIR MRI was performed at 7 Tesla to achieve high SNR and thus maximize sensitivity for detection of potential $\mathrm{A} \beta$ related tissue change. An automated parcellation algorithm was applied to PET- and MRI-volumes for topologic definition of brain structures, making possible to investigate regional distribution of $\mathrm{PiB}$ and FLAIR signals as well as their potential correlation.

\section{METHODS}

\section{RECRUITMENT AND PHENOTYPING OF THE STUDY COHORT}

Fourteen cognitively normal study participants aged between 60 and 79 years, without evidence for significant medical illness, were recruited as part of an ongoing study at our hospital (Steininger et al., 2014). Study procedures are in concordance with good clinical practice guidelines issued by the cantonal ethics committee Zürich, Switzerland and Swiss Federal Institute of Technology, respectively, (ETH Zürich), as well as with the declaration of Helsinki (World_Medical_Association, 1991).

In brief, normal cognitive performance levels of all participants was ascertained by psychiatric examination and neuropsychological testing including an initial screen for cognitive impairment [Mini Mental State Examination (MMSE); Folstein et al., 1975], followed by specific assessment of cognitive subdomains: Language skills were tested by applying the short version of the Boston Naming Test (BNT) from the CERADPlus testbattery (Nicholas et al., 1988; Thalmann et al., 1997); working memory performance was assessed by measuring memory span [digits forward and backward for short term memory assessment from the Wechsler Memory Scale-Revised (WMS-R) (Howard, 1950; Härting et al., 2000)]; cognitive flexibility was measured as an indicator of executive functioning [ratio of Trail Making Test A and B (Reitan, 1958; Tombaugh, 2004)]; memory performance was tested by applying the Verbal Learning and Memory Test (VLMT, immediate, delayed and supported recall) (Helmstaedter and Durwen, 1990; Helmstaedter, 2001). The VLMT is a modified german version of the auditory VLMT (Lezak, 1983; Müller et al., 1997). Medical history was assessed to exclude presence of significant medical illness in participants, complemented by Body mass index (BMI) as a general indicator of health (Mackay, 2010) (Table 1).

Exclusion criteria for the current study were: Cognitive deficits indicative for mild cognitive impairment (MCI) or dementia (Petersen et al., 1999; Winblad et al., 2004; Albert et al., 2011), significant medication or drug abuse with possible effects on cognition, general MRI exclusion criteria, contraindications against vein puncture, clinically relevant changes in red blood cell count, known allergy to the Carbon-11 based Pittsburgh Compund-B ( $\mathrm{PiB}$ ) positron emission tomography (PET) tracer or any of its constituents, history of severe allergic reactions to drugs or allergens, serious medical or neuropsychiatric illness and significant exposure to radiation, respectively.

\section{CARBON-11 BASED PITTSBURGH COMPOUND-B POSITRON EMISSION TOMOGRAPHY (PIB-PET) FOR ESTIMATION OF BRAIN A $\beta$}

Carbon-11 based Pittsburgh Compound-B for positron emission tomography (PiB-PET) based estimation of individual brain $\mathrm{A} \beta$ load (Mathis et al., 2003; Klunk et al., 2004; Solbach et al., 2005) was performed as reported earlier by our group at the PET Center of the Division of Nuclear Medicine, Zürich University Hospital utilizing a GE PET/CT Discovery scanner (Steininger et al., 2014). In brief, an individual dose of $350 \mathrm{MBq}$ of (11)carbon-labeled PiB was injected into the cubital vein. Images were corrected for attenuation using a low-dose CT. Standard quantitative filtered back projection algorithm including necessary corrections was applied.

Cerebral amyloid deposition values were extracted using a standard routine as implemented in PMOD Brain Tool software-package (PNEURO, Version 3.4, PMOD Technologies

\section{Table 1 | Demographics of the studied sample including} neuropsychological test results.

\begin{tabular}{lc}
\hline & Mean (SD) \\
\hline N (Females/Males) & $14(6 / 8)$ \\
Age (years) & $68.43(5.3)$ \\
Education (years) & $14.93(2.13)$ \\
Body Mass Index (BMI) & $25.83(3.9)$ \\
Cortical PiB retention & $1.23(0.34)$ \\
Mini Mental State Examination (MMSE) & $29.43(0.94)$ \\
Boston Naming Test (BNT) & $14.71(0.61)$ \\
Memory span, digits forward & $7.5(1.09)$ \\
Memory span, digits backward & $6.86(1.66)$ \\
Trail Making Test (ratio TMT-A by TMT-B) & $2.21(0.66)$ \\
VLMT: immediate recall & $11.43(2.31)$ \\
VLMT: delayed recall & $10.79(2.55)$ \\
VLMT: supported recall & $12(1.96)$ \\
\end{tabular}

Indicated are mean values with standard deviations (SD). 
Ltd, Zürich, Switzerland). Late frame (minutes 50-70) values were standardized by the cerebellar gray matter value, resulting in 3D-volumes of PiB-PET retention (matrix dimensions: $128 \times 128 \times 47$, voxel size: $2.34 \times 2.34 \times 3.27 \mathrm{~mm}$ ).

\section{MAGNETIC RESONANCE IMAGING (MRI) AT 7 TESLA}

MRI images were obtained on a Philips 7 Tesla Achieva whole-body scanner (Philips Healthcare, Best, The Netherlands) equipped with a Nova Medical quadrature transmit head coil and 32-channel receive coil array. 14 healthy elderly controls were scanned at the Institute for Biomedical Engineering (IBT) at the Swiss Federal Institute of Technology at Zürich, Switzerland (ETH Zürich). Acquired sequences included a high quality T1-weighted 3D MPRAGE sequence for structural brain image $[T E / T R=$ $3.74 \mathrm{~ms} / 8.12 \mathrm{~ms}$; scan mode: $3 \mathrm{D}$; total scan time: $654 \mathrm{~s}$; FOV (ap, $\mathrm{fh}, \mathrm{rl}): 220 \times 157.50 \times 199.38 \mathrm{~mm}$; resolution $(\mathrm{x}, \mathrm{y}, \mathrm{z}): 256 \times$ $260 \times 175$ ] for volumetric analysis of brain structures, and a $3 \mathrm{D}$ FLAIR sequence for assessment of regional tissue-edema $[T E / T R=310.74 / 8000 \mathrm{~ms}$; scan mode: $3 \mathrm{D}$; EPI $=1$; total scan time: $304 \mathrm{~s}$; FOV (ap, fh, rl): $220 \times 120 \times 200.87 \mathrm{~mm}$; scan resolution $(x, y, z): 368 \times 366 \times 60]$.

\section{STATISTICAL ANALYSIS OF MRI AND PiB-PET DATA}

T1 MPRAGE 3D volumes were postprocessed using an automated subcortical parcellation algorithm (Freesurfer image analysis suite; Fischl et al., 2004) for definition and volumetry of 29 cerebral anatomical structures included in the standard lookup table (FreeSurferColorLUT), as performed in earlier projects of our group (Unschuld et al., 2012a,b, 2013; Steininger et al., 2014). In a second step, Freesurfer image analysis suite was used for coregistration of FLAIR and PiB-PET volumes to the respective T1MPRAGE volume, allowing calculation of average intensity scores for each of the 29 brain regions of interest (ROIs) in each of the 14 participants (individual regional PiB-PET- and FLAIR intensity, respectively). All individual regional PiB-PET- and FLAIR intensity scores were normalized to the respective ROI-volume (PiB-PET/T1 and FLAIR/T1, respectively). Mean regional intensity scores were calculated for each of the 29 ROIs based on the respective 14 individual, volume normalized regional PiB-PETand FLAIR intensity scores, respectively. For subsequent statistical analysis, $z$-standardized intensity scores $(0=$ mean $)$ were obtained as follows $(z i=$ standardized intensity score; $i=$ rawvalue PiB-PET- and FLAIR intensity, respectively; $v=$ volume of the respective brain region in voxels; $\mu=$ arithmetic mean; $\sigma$ $=$ standard deviation $): z i=[(\mathrm{i} / \mathrm{v})-\mu] / \sigma$. For generation of standardized $\mathrm{z}$-scores reflecting variance between subjects for each region, $\mu$ and $\sigma$ were calculated for 29 samples, representing the assessed ROIs (individual regional PiB-PET- and FLAIR intensity). To assess general variance of regional PiB-PET- and FLAIR intensity, respectively, (mean regional PiB-PET- and FLAIR intensity scores), $\mu$ and $\sigma$ were calculated for 14 samples, representing the included study participants. Normalicy of mean regional intensities was tested by assuming a null hypothesis of normally distributed mean PiB-PET- and FLAIR intensity values for each region when applying Shapiro-Wilk test and Q-Q plots, as well as Levene's test for homogeneity of variances (IBM SPSS Statistics, Armonk, NY, USA, Version 20.0). Statistical dependence was tested, assuming a null hypothesis of independent regional PiBPET- and FLAIR intensity scores, using Pearson's correlation analysis $(r)$. The MatLab software package [The MathWorks, Inc., Natick, MA, USA, Version 8.3.0.532 (R2014a)] with Statistics Toolbox (Version 9.0) and Symbolic Math Toolbox (Version 6.0) were used to investigate each of the 29 ROIs for correlations between regional PiB-PET- and FLAIR intensity of each participant $(n=14)$ using non-parametric Spearman's rank correlation (rho). To account for multiplicity bias, a correction for multiple testing according to Holm-Bonferroni was applied to $p$-values resulting from the 29 Spearman's rank correlation tests (Holm, 1979).

\section{RESULTS \\ NEUROPSYCHOLOGICAL ASSESSMENT INDICATES NORMAL TEST PERFORMANCE OF STUDY POPULATION}

MMSE did not reveal evidence for cognitive impairment in the study population, as indicated by group-average [standard deviation (SD)] test score of 29.43 (0.94). Consistently, neuropsychological assessment indicated individual test performances within the normal range: Average performance in the BNT was 14.71 (0.61); Memory Span digits forward 7.5 (1.09), digits backward 6.86 (1.66); Trail Making Test ratio A by B: 2.24 (0.67) and results of the VLMT (immediate, delayed, and supported recall, respectively) were 11.43 (2.31), 10.79 (2.55), 12.0 (1.96). Mean age of the studied population was 68 years [ $(S D) 5]$ years, mean time of education was 14.93 years (SD 2.13). Mean BMI was 25.83 (SD 3.90) (Table 1).

\section{MEAN REGIONAL PIB RETENTION SCORES AND FLAIR INTENSITIES ARE NORMALLY DISTRIBUTED AND INDEPENDENT}

Twenty-nine brain regions were defined by automated anatomical labeling, making possible estimation of regional volumes based on the T1-MR-data and respective average PiB retention scores and FLAIR intensities, respectively. All PiB-PET and FLAIR intensities were normalized to the respective regional volumes based on the T1-image (Table 2) and converted to standardized z-scores. Tests of normality indicated normally distributed average regional PiB retention $(d f=29$, Shapiro-Wilk $=0.95, p=0.16)$ and FLAIR intensities $(d f=29$, ShapiroWilk $=0.99, p=0.95)$. Homogeneity of variances $(\sigma 2)$ of mean regional FLAIR and PiB-PET intensities was indicated by nonsignificant Levene's Test $[\sigma 2($ FLAIR $)=0.57, \sigma 2($ PiB-PET $)=$ $0.55, p=0.937$ ] (Figure 1). No evidence of statistical dependence between regional average $\mathrm{PiB}$ retention and FLAIR intensity could be observed when Pearson's correlation analysis was performed $(r=-0.18 ; p=0.35)$. Ranking of average PiB-PET values by size resulted in highest relative $\mathrm{PiB}$ retention for Pallidum [1.34 (0.08)], right ventral Diencephalon $[1.17(0.12)]$ and Brainstem $[1.12(0.15)]$. Lowest values resulted for Optic Chiasm $[-1.99$ (0.16)], left Accumbens Area $[-0.79(0.16)]$, and left Choroid Plexus [-0.72 (0.17)] (Figure 2A). Highest mean regional FLAIR intensities were observable for right Amygdala [1.52 (0.11)], left Amygdala [1.36 (0.11)], and left Accumbens area [1.07 (0.11)]. Lowest values resulted for left Pallidum $[-1.53(0.13)]$, right Pallidum $[-1.36(0.10)]$, and left Choroid Plexus $[-1.15(0.16)]$ (Figure 2B). 
Table 2 | Volumes of brain structures as derived from the 7T T1 MPRAGE images as well as mean regional PiB-PET and FLAIR intensities, normalized to volume.

\begin{tabular}{|c|c|c|c|c|c|c|}
\hline ROI & $\begin{array}{c}\text { Volume T1 (ml) } \\
\text { mean (s.e.m.) }\end{array}$ & FLAIR/T1 & $\mathrm{PiB} / \mathrm{T1}$ & \multicolumn{3}{|c|}{ Spearman correlation FLAIR/T1 with PiB/T1 } \\
\hline Brain-stem & $19.8(0.6)$ & $6.58(0.53)$ & $0.83(0.03)$ & 0.85 & 3.56 & *0.0076 \\
\hline Left-hippocampus & $2.84(0.48)$ & $62.57(13.5)$ & $7.14(1.18)$ & 0.84 & 3.41 & *0.0105 \\
\hline Left-vessel (basal ganglia) & $0.11(0.02)$ & $1539.76(280.43)$ & $203.79(53.32)$ & 0.82 & 2.90 & *0.0324 \\
\hline Right-vessel (basal ganglia) & $0.16(0.02)$ & $1211.58(268.79)$ & $120.21(21.94)$ & 0.69 & 1.86 & 0.33 \\
\hline Right-ventral DC & $2.95(0.12)$ & $37.17(3.6)$ & $5.69(0.27)$ & 0.65 & 1.56 & 0.63 \\
\hline Right-caudate & $3.31(0.48)$ & $40.22(6.1)$ & $4.77(0.57)$ & 0.64 & 1.56 & 0.6 \\
\hline Right-accumbens-area & $0.45(0.07)$ & $364.38(64.34)$ & $38.44(8.25)$ & 0.61 & 1.41 & 0.82 \\
\hline Right-amygdala & $1.29(0.12)$ & $126.13(14.14)$ & $11.12(0.95)$ & 0.60 & 1.32 & 0.95 \\
\hline Left-accumbens-area & $0.52(0.05)$ & $296.24(33.2)$ & $30.17(8.95)$ & 0.57 & 1.16 & 0.99 \\
\hline Right-pallidum & $1.29(0.07)$ & $68.34(8.53)$ & $13.84(0.82)$ & 0.49 & 0.83 & 0.99 \\
\hline CC_Posterior & $0.94(0.03)$ & $105(10.23)$ & $16.6(0.73)$ & 0.43 & 0.59 & 0.99 \\
\hline CC_Central & $0.36(0.02)$ & 335.93 (34.28) & $35.2(1.58)$ & 0.39 & 0.47 & 0.99 \\
\hline Left-ventral DC & $3.09(0.15)$ & $33.79(3.31)$ & $5.39(0.29)$ & 0.36 & 0.39 & 0.99 \\
\hline Left-thalamus-proper & $6.29(1.04)$ & $18.29(2.12)$ & $2.63(0.2)$ & 0.35 & 0.36 & 0.99 \\
\hline Right-putamen & $4.62(0.38)$ & $27.35(3.27)$ & $3.52(0.29)$ & 0.34 & 0.32 & 0.99 \\
\hline Left-caudate & $2.48(0.14)$ & $48.99(6.66)$ & $5.25(0.5)$ & 0.29 & 0.20 & 0.99 \\
\hline Right-thalamus-proper & $6.1(0.58)$ & $19.59(1.92)$ & $2.51(0.2)$ & 0.26 & 0.14 & 0.99 \\
\hline CC_Mid_Posterior & $0.33(0.02)$ & 348.89 (35.27) & $37.26(1.76)$ & 0.25 & 0.11 & 0.99 \\
\hline Right-cerebral-cortex & $152.89(4.28)$ & $0.78(0.05)$ & $0.09(0.01)$ & 0.12 & 0.00 & 0.99 \\
\hline Left-cerebral-cortex & 176.77 (9.06) & $0.69(0.06)$ & $0.08(0.01)$ & 0.10 & 0.00 & 0.99 \\
\hline
\end{tabular}

Labels refer to anatomical ROIs defined by the FreeSurfer whole brain segmentation algorithm; DC, diencephalon; CC, corpus callosum. Indicated are mean values with standard errors of the mean (SEM), as well as statistical dependence between regional PiB-PET and FLAIR intensities [Spearman rank correlation coefficients (rho), significant relationships at $p<0.05$ after correction for multiple testing are indicated by "*"].

\section{INDIVIDUAL PIB RETENTION SCORES AND FLAIR INTENSITY SIGNIFICANTLY CORRELATE FOR BRAIN REGIONS INCLUDING HIPPOCAMPUS, BRAINSTEM, AND BASAL GANGLIA VESSELS}

To identify region-specific relationships between $A \beta$-deposition and FLAIR intensity, for each of the 29 investigated brain regions a Spearman's correlation coefficients were calculated based on individual regional PiB-PET- and FLAIR intensity, as measured in each of the 14 participants. For 10 out of 29 brain regions a nominally significant relationship could be observed: Right Hippocampus (rho = $0.86,-\log (\mathrm{p})=3.84)$, Brainstem $($ rho $=0.85,-\log (\mathrm{p})=$ 3.56), left Hippocampus ( $\mathrm{rho}=0.84,-\log (\mathrm{p})=3.41$ ), left Basal Ganglia vessels ( $r h o=0.82,-\log (\mathrm{p})=2.90$ ), left Choroid Plexus (rho $=0.73,-\log (\mathrm{p})=2.05$ ), right Basal Ganglia vessels (rho $=0.69,-\log (\mathrm{p})=1.86$ ), right ventral Diencephalon (rho $=0.65,-\log (\mathrm{p})=1.56)$, right Caudate $(\mathrm{rho}=0.64,-\log (\mathrm{p})=1.56)$, right Accumbens area
$($ rho $=0.61,-\log (\mathrm{p})=1.41)$, and right Amygdala (rho $=$ $0.60,-\log (\mathrm{p})=1.32$ ) (Table 2 and Figure 3A). When applying correction for multiple testing using the Holm-Bonferroni method (Holm, 1979), four regions remained significant: Right Hippocampus $(p=0.0042)$, Brainstem $(p=0.0076)$, left Hippocampus $(p=0.011)$, left Basal Ganglia vessels $(p=0.32)$ (Table 2 and Figure 3B).

\section{DISCUSSION}

Our data indicate a significant relationship between regional $\mathrm{A} \beta$ - accumulation, as measured by PiB-PET, and tissue-edema, as indicated by FLAIR intensity, in the hippocampus, brainstem and basal ganglia vessel region of cognitively normal elderly adults. While, to our knowledge, this is the first study to apply FLAIR MRI at high magnetic field strength of 7 Tesla for investigation of $A \beta$ associated brain change, our findings are consistent with earlier reports on subcortical and limbic 

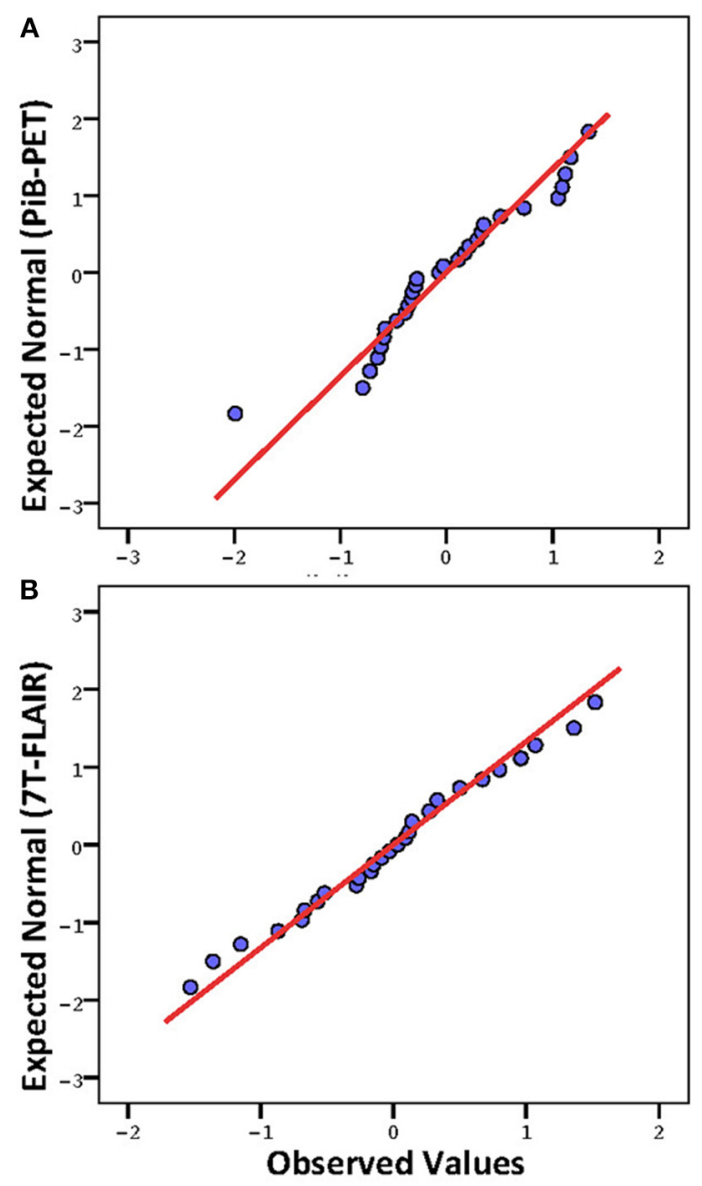

FIGURE 1 | Q-Q Plots of mean regional intensities (standardized z-scores; $\mathbf{0}=$ mean, values indicate standard deviations from mean).

(A) Pittsburgh Compund-B positron emission tomography; (B)

Fluid-attenuated inversion recovery (FLAIR) 7 Tesla MRI (7T-FLAIR).

nuclei being particularly sensitive to age-related neurodegenerative pathology.

PiB-PET is a well established neuroimaging method for measuring brain $\mathrm{A} \beta$ deposition in elderly subjects with increased risk for $\mathrm{AD}$ and to investigate brain change associated with $\mathrm{A} \beta-$ accumulation (Klunk et al., 2004; Jack et al., 2009; Mormino et al., 2009; Steininger et al., 2014). PiB-PET studies on populations with sporadic $\mathrm{AD}$ are consistent with neuropathological data, as they indicate $A \beta$-accumulation spreading from the neocortex to the entire brain (Braak and Braak, 1991; Serrano-Pozo et al., 2011; Jack and Holtzman, 2013). Preclinical stages in individuals with genetic predisposition for familial $\mathrm{AD}$ however, appear to rather be characterized by PiB retention in striatal regions (Klunk et al., 2004; Bateman et al., 2012). A recent study investigating a large sample of cognitively normal elderly subjects showed that subtle increases of local $\mathrm{A} \beta$ indicate significant hypometabolism in $\mathrm{AD}$ signature regions including angular gyrus, posterior cingulate and temporal lobe (Lowe et al., 2014). Moreover, an earlier study reports correlations between increased $\mathrm{A} \beta$-levels in temporal neocortex and posterior cingulate cortex of cognitively normal elderly with accelerated cortical atrophy (Chetelat et al., 2012). These reports are consistent with the observation of significant spatial variation of $A \beta$-deposition between brain regions (Price et al., 2005; Mintun et al., 2006; Su et al., 2013) and highlight significance of effects associated with $\mathrm{A} \beta$-load in distinct brain regions for progression of age-related neurodegeneration. In the current study, region-specific investigation of $A \beta$-associated brain-change was performed using whole brain segmentation tools provided by the FreeSurfer software package, as demonstrated earlier to provide high reliability for analysis of quantitative PiB-PET data (Fischl et al., 2004; Su et al., 2013): By defining brain ROIs, volumes were determined based on structural T1-MRI data as well as respective intensities for PiB retention and FLAIR contrast. The resulting regional average PiB-PET and FLAIR intensities were normally distributed and independent, thus minimizing probability of bias by brain region-specific variations of sensitivity of either of the two contrasts applied. Each brain region was tested for correlations between individual PiB-PET and FLAIR intensities using Spearman's correlation coefficient as a non-parametric test allowing for the relatively small sample size (Bonett and Wright, 2000), followed by correction for multiple testing (Holm, 1979). In doing so, significant relationships between PiB-PET and FLAIR intensities could be observed for right and left Hippocampus, Brainstem and also a small region including left Basal Ganglia vessels. As the FLAIR contrast reflects a wide spectrum of pathological brain-tissue alterations associated with regional edema (Young et al., 2008; Neema et al., 2009; Carlson et al., 2011), our finding is consistent with earlier reports on signature-regions of AD primarily affected by age-related neurodegeneration: The Hippocampus has been shown by numerous studies to be particularly sensitive to aging related brain change and AD in particular (de Leon et al., 1989; Frisoni et al., 2011; Serrano-Pozo et al., 2011) and neurodegenerative processes can be observed in gray matter nuclei located in Brainstem and Basal Ganglia (Iseki et al., 1989; Parvizi et al., 2001; Simic et al., 2009; Braak and Del Tredici, 2012; Brothers et al., 2013). Notably, significant relationships with FLAIR intensity were not determined by brain regions with highest $\mathrm{PiB}$ retention, which may support considerations on pathological relevance of subtle increases of $\mathrm{A} \beta$ in vulnerable brain regions (Mormino et al., 2012; Lowe et al., 2014), potentially mediated by additional factors that may determine resilience of distinct neuronal populations (Steffener and Stern, 2012). Our data appear consistent with earlier reports on a relationship between FLAIR hyperintensity and cerebral $A \beta$-burden, as FLAIR intensity of white matter regions has been shown to predict progression of $A \beta$-accumulation, thus potentially representing a risk factor for neurodegeneration and AD (Grimmer et al., 2012). However, focal tissue-edema in the brain, as indicated by FLAIR hyperintensity, may also be observed during treatment with antibodies targeted against $A \beta$, thus potentially reflecting tissue processes associated with clearance of $A \beta$ (Frisoni, 2012; Sperling et al., 2012).

Limitations of the current study include the fact that while SNR of the FLAIR sequence significantly benefit from high field strengths (Visser et al., 2010; Zwanenburg et al., 2010) and sensitivity for detection of subtle changes thus may have been increased by using FLAIR MRI at 7 Tesla, findings nevertheless need to be treated with caution, as clinical relevance of the 

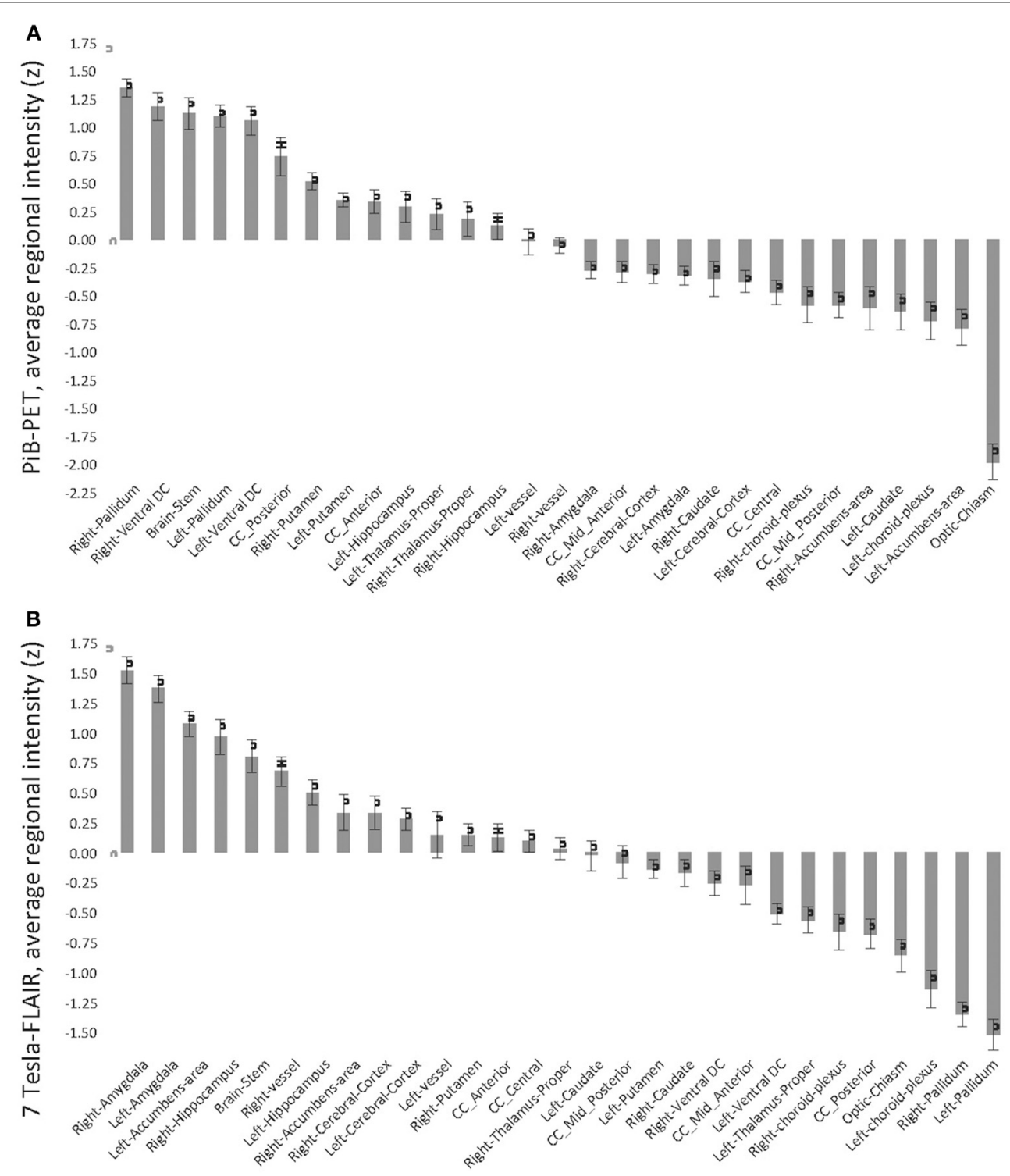

FIGURE 2 | Standardized z-scores of regional average intensities, normalized by T1-volume, sorted in descending order. (A) Pittsburgh Compund-B positron emission tomography; (B) Fluid-attenuated inversion recovery (FLAIR) 7 Tesla MRI (7T-FLAIR).

increased sensitivity has not been tested. While FLAIR MRI so far has been used mainly for qualitative visual assessment of brain tissue abnormalities (De Coene et al., 1992; Adams and Melhem, 1999), ROI-based quantification of FLAIR signal intensity has been performed recently for investigation of brain pathology in a context of acute stroke (Cheng et al., 2013). Nevertheless, as FLAIR has limited capacities for quantification of single voxelintensities and MR-sequences implementing T2-relaxometry may provide a better quantitative measure, this also needs to be considered as a potential limitation of the here performed approach of correlating FLAIR intensities with PiB retention (Pell et al., 2004; Deoni, 2010; Cheng et al., 2012). Another limitation is the fact that as for the current study a cross-sectional design was applied, no prospective inferences can be made regarding effects of our findings on participant's risk for AD. Moreover, as high brainstem $\mathrm{PiB}$ uptake has been shown to indicate $A \beta$ in Parkinson's disease with dementia (Maetzler et al., 2008), our findings might also reflect brain change in a context of other neurodegenerative pathologies than $\mathrm{AD}$.

Taken together, our finding of a region specific correlation between PiB retention, indicating $A \beta$-accumulation, and FLAIR hyperintensities in cognitively normal elderly subjects is consistent with earlier reports on $A \beta$-associated brain change taking place decades before manifestation of $\mathrm{AD}$ as well as signatureregions for neurodegenerative dementia in general. Additional longitudinal studies are needed to clarify whether our findings reflect changes associated with increased risk for age-related brain disease or rather may indicate compensatory brain change, 


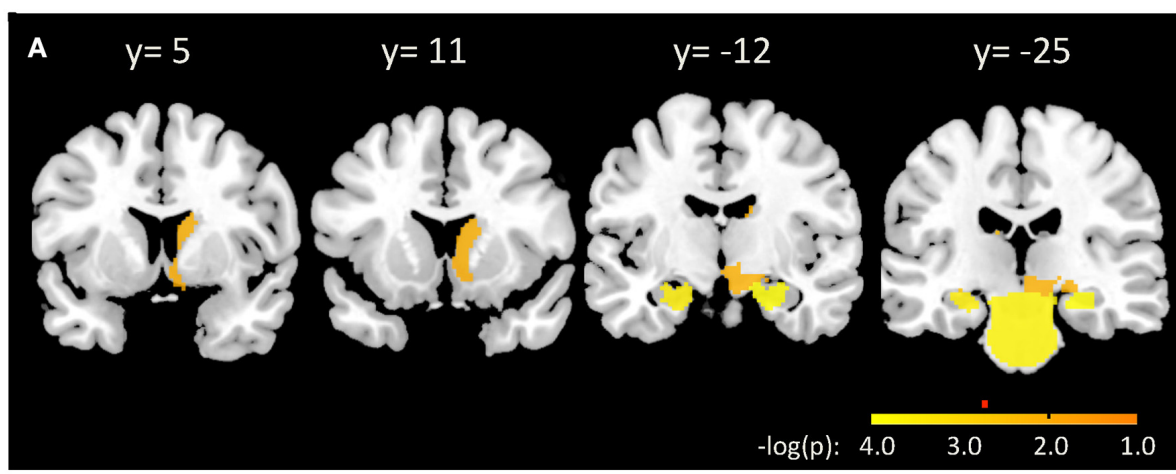

B

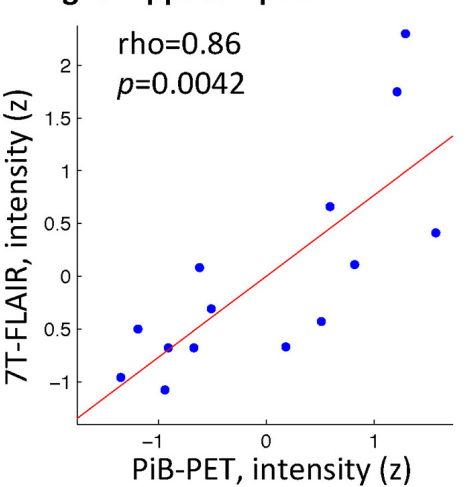

Left Hippocampus

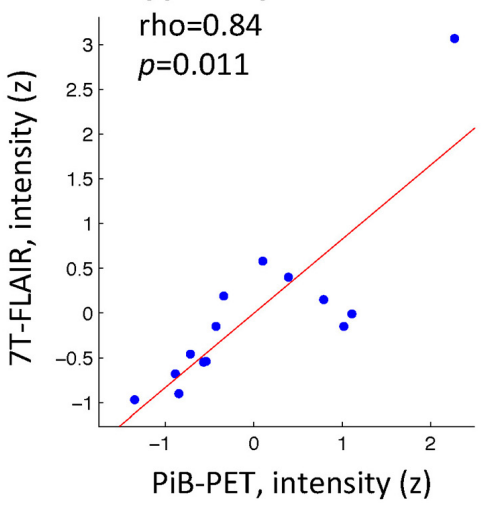

FIGURE 3 | (A) Relationship between individual regional PiB-PET and 7T FLAIR intensities as indicated by spearman correlation analysis. Displayed are regions with $-\log (p)>1.3$. Alpha $=0.05$ after correction for multiple testing for ROls with $-\log (p)>2.7$, as indicated by red marker. Y-positions refer to
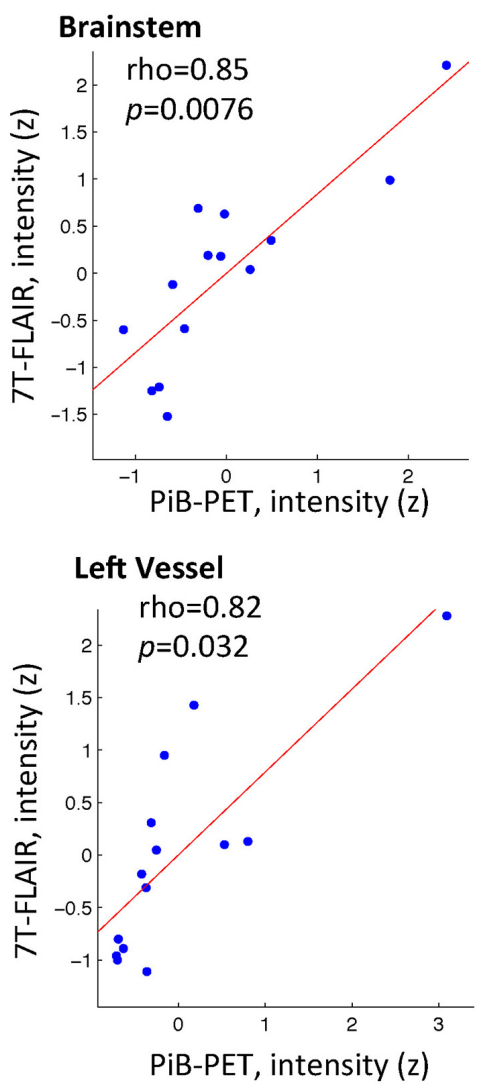

MNI space. (B) Brain regions with strongest relationship between individual regional PiB-PET and 7T FLAIR, as indicated by significant spearman correlation after correction of $p$-values for multiple testing

(Bonferroni-Holms). Each study-participant is represented by one dot. resulting in normal cognitive performance despite prevalent $A \beta$ burden.

\section{ACKNOWLEDGMENTS}

We thank all subjects for their study participation. We thank Linjing $\mathrm{Mu}, \mathrm{PhD}$ and Geoff Warnock, $\mathrm{PhD}$ from the Division of Nuclear Medicine, University of Zürich, Switzerland for their help in generation of (11)carbon-labeled Pittsburgh Compound$\mathrm{B}$ tracer for positron emission tomography (Linjing $\mathrm{Mu}$ ) and calculation of the cortical PiB retention scores (Geoff Warnock).
We thank Daniel Summermatter from Division of Psychiatry Research and Psychogeriatric Medicine, University of Zürich, Switzerland, for help in interpretation of neuropsychological test results. This work was funded by the Molecular Imaging Network Zürich (MINZ), the Swiss National Science Foundation (Schweizerischer Nationalfonds, SNF), institutional support from the Division of Psychiatry Research and Psychogeriatric Medicine, University of Zürich and Institute for Biomedical Engineering, University of Zürich and ETH Zürich, Zürich, Switzerland. 


\section{REFERENCES}

Adams, J. G., and Melhem, E. R. (1999). Clinical usefulness of T2-weighted fluidattenuated inversion recovery MR imaging of the CNS. AJR Am. J. Roentgenol. 172, 529-536. doi: 10.2214/ajr.172.2.9930818

Albert, M. S., Dekosky, S. T., Dickson, D., Dubois, B., Feldman, H. H., Fox, N. C., et al. (2011). The diagnosis of mild cognitive impairment due to Alzheimer's disease: recommendations from the National Institute on AgingAlzheimer's Association workgroups on diagnostic guidelines for Alzheimer's disease. Alzheimers Dement. 7, 270-279. doi: 10.1016/j.jalz.2011.03.008

Alzheimer, A. (1907). Über eine eigenartige Erkrankung der Hirnrinde. Allgemeine Zeitschrift für Psychiatrie und Psychiatrisch-Gerichtliche Medizin 64, 146-148.

Bateman, R. J., Xiong, C., Benzinger, T. L., Fagan, A. M., Goate, A., Fox, N. C., et al. (2012). Clinical and biomarker changes in dominantly inherited Alzheimer's disease. N. Engl. J. Med. 367, 795-804. doi: 10.1056/NEJMoa1202753

Bonett, D. G., and Wright, T. A. (2000). Sample size requirements for estimating Pearson, Kendall and Spearman correlations Psychometrika 65, 23-28. doi: 10.1007/BF02294183

Braak, H., and Braak, E. (1991). Neuropathological stageing of Alzheimer-related changes. Acta Neuropathol. 82, 239-259. doi: 10.1007/BF00308809

Braak, H., and Del Tredici, K. (2012). Where, when, and in what form does sporadic Alzheimer's disease begin? Curr. Opin. Neurol. 25, 708-714. doi: 10.1097/WCO.0b013e32835a3432

Brothers, H. M., Bardou, I., Hopp, S. C., Marchalant, Y., Kaercher, R. M., Turner, S. M., et al. (2013). Time-dependent compensatory responses to chronic neuroinflammation in hippocampus and brainstem: the potential role of glutamate neurotransmission. J. Alzheimers. Dis. Parkinsonism 3:110. doi: 10.4172/21610460.1000110

Carlson, C., Estergard, W., Oh, J., Suhy, J., Jack, C. R. Jr., Siemers, E., et al. (2011). Prevalence of asymptomatic vasogenic edema in pretreatment Alzheimer's disease study cohorts from phase 3 trials of semagacestat and solanezumab. Alzheimers. Dement. 7, 396-401. doi: 10.1016/j.jalz.2011.05.2353

Cheng, B., Brinkmann, M., Forkert, N. D., Treszl, A., Ebinger, M., Kohrmann, M., et al. (2013). Quantitative measurements of relative fluid-attenuated inversion recovery (FLAIR) signal intensities in acute stroke for the prediction of time from symptom onset. J. Cereb. Blood Flow Metab. 33, 76-84. doi: 10.1038/jcbfm.2012.129

Cheng, H. L., Stikov, N., Ghugre, N. R., and Wright, G. A. (2012). Practical medical applications of quantitative MR relaxometry. J. Magn. Reson. Imaging 36, 805-824. doi: 10.1002/jmri.23718

Chetelat, G., Villemagne, V. L., Villain, N., Jones, G., Ellis, K. A., Ames, D., et al. (2012). Accelerated cortical atrophy in cognitively normal elderly with high beta-amyloid deposition. Neurology 78, 477-484. doi: 10.1212/WNL.0b013e318246d67a

De Coene, B., Hajnal, J. V., Gatehouse, P., Longmore, D. B., White, S. J., Oatridge, A., et al. (1992). MR of the brain using fluid-attenuated inversion recovery (FLAIR) pulse sequences. AJNR Am. J. Neuroradiol. 13, 1555-1564.

de Leon, M. J., George, A. E., Stylopoulos, L. A., Smith, G., and Miller, D. C. (1989). Early marker for Alzheimer's disease: the atrophic hippocampus. Lancet 2, 672-673. doi: 10.1016/S0140-6736(89)90911-2

Deoni, S. C. (2010). Quantitative relaxometry of the brain. Top. Magn. Reson. Imaging 21, 101-113. doi: 10.1097/RMR.0b013e31821e56d8

Fischl, B., Salat, D. H., Van Der Kouwe, A. J., Makris, N., Segonne, F., Quinn, B. T., et al. (2004). Sequence-independent segmentation of magnetic resonance images. Neuroimage 23(Suppl. 1), S69-S84. doi: 10.1016/j.neuroimage.2004.07.016

Folstein, M. F., Folstein, S. E., and McHugh, P. R. (1975). “Mini-mental state.” A practical method for grading the cognitive state of patients for the clinician. J. Psychiatr. Res. 12, 189-198. doi: 10.1016/0022-3956(75)90026-6

Frisoni, G. B. (2012). ARIA from off-key operas? Lancet Neurol. 11, 207-208. doi: 10.1016/S1474-4422(12)70021-2

Frisoni, G. B., Winblad, B., and O'Brien, J. T. (2011). Revised NIA-AA criteria for the diagnosis of Alzheimer's disease: a step forward but not yet ready for widespread clinical use. Int. Psychogeriatr. 23, 1191-1196. doi: $10.1017 /$ S1041610211001220

Grimmer, T., Faust, M., Auer, F., Alexopoulos, P., Forstl, H., Henriksen, G., et al. (2012). White matter hyperintensities predict amyloid increase in Alzheimer's disease. Neurobiol. Aging 33, 2766-2773. doi: 10.1016/j.neurobiolaging.2012.01.016
Härting, C., Markowitsch, H. J., Neufeld, H., Calabrese, P., Diesinger, K., and Kessler, J. (2000). Wechsler Gedächtnis Test - Revidierte Fassung (WMS-R). Bern: Huber.

Helmstaedter, C. (2001). Verbaler Lern- und Merkfähigkeitstest: VLMT, Manual. Gottingen: Beltz Test GmbH.

Helmstaedter, C., and Durwen, H. F. (1990). VLMT: Verbaler Lern- und Merkfähigkeitstest [VLMT: Verbal learning and memory test]. Schweizer Archiv für Neurologie und Psychiatrie 141, 21-30.

Hock, C., and Nitsch, R. M. (2000). [Alzheimer dementia]. Praxis 89, 529-540.

Holm, S. (1979). A simple sequentially rejective bonferroni test procedure. Scand. J. Stat. 6, 65-70.

Howard, A. R. (1950). Diagnostic value of the Wechsler Memory Scale with selected groups of institutionalized patients. J. Consult. Psychol. 14, 376-380. doi: 10.1037/h0058479

Iacono, D., O’Brien, R., Resnick, S. M., Zonderman, A. B., Pletnikova, O., Rudow, G., et al. (2008). Neuronal hypertrophy in asymptomatic Alzheimer disease. J. Neuropathol. Exp. Neurol. 67, 578-589. doi: 10.1097/NEN.0b013e3181772794

Iseki, E., Matsushita, M., Kosaka, K., Kondo, H., Ishii, T., and Amano, N. (1989). Distribution and morphology of brain stem plaques in Alzheimer's disease. Acta Neuropathol. 78, 131-136. doi: 10.1007/BF00688200

Jack, C. R. Jr., and Holtzman, D. M. (2013). Biomarker modeling of Alzheimer's disease. Neuron 80, 1347-1358. doi: 10.1016/j.neuron.2013.12.003

Jack, C. R. Jr., Lowe, V. J., Weigand, S. D., Wiste, H. J., Senjem, M. L., Knopman, D. S., et al. (2009). Serial PIB and MRI in normal, mild cognitive impairment and Alzheimer's disease: implications for sequence of pathological events in Alzheimer's disease. Brain 132, 1355-1365. doi: 10.1093/brain/awp062

Klunk, W. E., Engler, H., Nordberg, A., Wang, Y., Blomqvist, G., Holt, D. P., et al. (2004). Imaging brain amyloid in Alzheimer's disease with Pittsburgh Compound-B. Ann. Neurol. 55, 306-319. doi: 10.1002/ana.20009

Knopman, D. S., Parisi, J. E., Salviati, A., Floriach-Robert, M., Boeve, B. F., Ivnik, R. J., et al. (2003). Neuropathology of cognitively normal elderly. J. Neuropathol. Exp. Neurol. 62, 1087-1095.

Lezak, M. D. (1983). Neuropsychological Assessment, 2nd Edn. New York, NY: Oxford University Press.

Lowe, V. J., Weigand, S. D., Senjem, M. L., Vemuri, P., Jordan, L., Kantarci, K., et al. (2014). Association of hypometabolism and amyloid levels in aging, normal subjects. Neurology 82, 1959-1967. doi: 10.1212/WNL.0000000000000467

Mackay, N. J. (2010). Scaling of human body mass with height: the body mass index revisited. J. Biomech. 43, 764-766. doi: 10.1016/j.jbiomech.2009.10.038

Maetzler, W., Reimold, M., Liepelt, I., Solbach, C., Leyhe, T., Schweitzer, K., et al. (2008). [11C]PIB binding in Parkinson's disease dementia. Neuroimage 39, 1027-1033. doi: 10.1016/j.neuroimage.2007.09.072

Mathis, C. A., Wang, Y., Holt, D. P., Huang, G. F., Debnath, M. L., and Klunk, W. E. (2003). Synthesis and evaluation of 11C-labeled 6-substituted 2arylbenzothiazoles as amyloid imaging agents. J. Med. Chem. 46, 2740-2754. doi: $10.1021 / \mathrm{jm} 030026 \mathrm{~b}$

Mintun, M. A., Larossa, G. N., Sheline, Y. I., Dence, C. S., Lee, S. Y., Mach, R. H., et al. (2006). [11C]PIB in a nondemented population: potential antecedent marker of Alzheimer disease. Neurology 67, 446-452. doi: 10.1212/01.wnl.0000228230.26044.a4

Mormino, E. C., Brandel, M. G., Madison, C. M., Rabinovici, G. D., Marks, S., Baker, S. L., et al. (2012). Not quite PIB-positive, not quite PIB-negative: slight PIB elevations in elderly normal control subjects are biologically relevant. Neuroimage 59, 1152-1160. doi: 10.1016/j.neuroimage.2011.07.098

Mormino, E. C., Kluth, J. T., Madison, C. M., Rabinovici, G. D., Baker, S. L., Miller, B. L., et al. (2009). Episodic memory loss is related to hippocampalmediated beta-amyloid deposition in elderly subjects. Brain 132, 1310-1323. doi: 10.1093/brain/awn320

Müller, H., Hasse-Sander, I., Horn, R., Helmstaedter, C., and Elger, C. E. (1997). Rey Auditory-Verbal Learning Test: structure of a modified German version. J. Clin. Psychol. 53, 663-671.

Neema, M., Guss, Z. D., Stankiewicz, J. M., Arora, A., Healy, B. C., and Bakshi, R. (2009). Normal findings on brain fluid-attenuated inversion recovery MR images at 3T. AJNR Am. J. Neuroradiol. 30, 911-916. doi: 10.3174/ajnr. A1514

Nicholas, L. E., Brookshire, R. H., MacLennan, D. L., Schumacher, J. G., and Porrazzo, S. A. (1988). The boston naming test: revised administration and scoring procedures and normative information for non-brain-damaged adults. Clin. Aphasiol. 18, 103-115. 
Parvizi, J., Van Hoesen, G. W., and Damasio, A. (2001). The selective vulnerability of brainstem nuclei to Alzheimer's disease. Ann. Neurol. 49, 53-66. doi: 10.1002/1531-8249(200101)49:1\%3C53::AID-ANA30\%3E3.0.CO;2-Q

Pell, G. S., Briellmann, R. S., Waites, A. B., Abbott, D. F., and Jackson, G. D. (2004). Voxel-based relaxometry: a new approach for analysis of T2 relaxometry changes in epilepsy. Neuroimage 21, 707-713. doi: 10.1016/j.neuroimage.2003.09.059

Petersen, R. C., Smith, G. E., Waring, S. C., Ivnik, R. J., Tangalos, E. G., and Kokmen, E. (1999). Mild cognitive impairment: clinical characterization and outcome. Arch. Neurol. 56, 303-308. doi: 10.1001/archneur.56.3.303

Price, J. C., Klunk, W. E., Lopresti, B. J., Lu, X., Hoge, J. A., Ziolko, S. K., et al. (2005). Kinetic modeling of amyloid binding in humans using PET imaging and Pittsburgh Compound-B. J. Cereb. Blood Flow Metab. 25, 1528-1547. doi: 10.1038/sj.jcbfm.9600146

Price, J. L., and Morris, J. C. (1999). Tangles and plaques in nondemented aging and "preclinical" Alzheimer's disease. Ann. Neurol. 45, 358-368.

Reitan, R. M. (1958). Validity of the Trail Making Test as an indicator of organic brain damage. Percept. Mot. Skills 8, 271-276. doi: 10.2466/pms.1958.8.3.271

Riudavets, M. A., Iacono, D., Resnick, S. M., O’Brien, R., Zonderman, A. B., Martin, L. J., et al. (2007). Resistance to Alzheimer's pathology is associated with nuclear hypertrophy in neurons. Neurobiol. Aging 28, 1484-1492. doi: 10.1016/j.neurobiolaging.2007.05.005

Rodrigue, K. M., Kennedy, K. M., Devous, M. D. Sr., Rieck, J. R., Hebrank, A. C., Diaz-Arrastia, R., et al. (2012). beta-Amyloid burden in healthy aging: regional distribution and cognitive consequences. Neurology 78, 387-395. doi: 10.1212/WNL.0b013e318245d295

Savva, G. M., Wharton, S. B., Ince, P. G., Forster, G., Matthews, F. E., Brayne, C., et al. (2009). Age, neuropathology, and dementia. N. Engl. J. Med. 360, 2302-2309. doi: 10.1056/NEJMoa0806142

Serrano-Pozo, A., Frosch, M. P., Masliah, E., and Hyman, B. T. (2011). Neuropathological alterations in Alzheimer disease. Cold Spring Harb. Perspect. Med. 1:a006189. doi: 10.1101/cshperspect.a006189

Sheline, Y. I., Raichle, M. E., Snyder, A. Z., Morris, J. C., Head, D., Wang, S., et al. (2010). Amyloid plaques disrupt resting state default mode network connectivity in cognitively normal elderly. Biol. Psychiatry 67, 584-587. doi: 10.1016/j.biopsych.2009.08.024

Simic, G., Stanic, G., Mladinov, M., Jovanov-Milosevic, N., Kostovic, I., and Hof, P. R. (2009). Does Alzheimer's disease begin in the brainstem? Neuropathol. Appl. Neurobiol. 35, 532-554. doi: 10.1111/j.1365-2990.2009.01038.x

Solbach, C., Uebele, M., Reischl, G., and Machulla, H. J. (2005). Efficient radiosynthesis of carbon-11 labelled uncharged Thioflavin $\mathrm{T}$ derivatives using [11C]methyl triflate for beta-amyloid imaging in Alzheimer's disease with PET. Appl. Radiat. Isot. 62, 591-595. doi: 10.1016/j.apradiso.2004.09.003

Sperling, R. A., Aisen, P. S., Beckett, L. A., Bennett, D. A., Craft, S., Fagan, A. M., et al. (2011). Toward defining the preclinical stages of Alzheimer's disease: recommendations from the National Institute on Aging-Alzheimer's Association workgroups on diagnostic guidelines for Alzheimer's disease. Alzheimers Dement. 7, 280-292. doi: 10.1016/j.jalz.2011.03.003

Sperling, R. A., Johnson, K. A., Doraiswamy, P. M., Reiman, E. M., Fleisher, A. S., Sabbagh, M. N., et al. (2013). Amyloid deposition detected with florbetapir F 18 ((18)F-AV-45) is related to lower episodic memory performance in clinically normal older individuals. Neurobiol. Aging 34, 822-831. doi: 10.1016/j.neurobiolaging.2012.06.014

Sperling, R., Salloway, S., Brooks, D. J., Tampieri, D., Barakos, J., Fox, N. C., et al. (2012). Amyloid-related imaging abnormalities in patients with Alzheimer's disease treated with bapineuzumab: a retrospective analysis. Lancet Neurol. 11, 241-249. doi: 10.1016/S1474-4422(12)70015-7

Steffener, J., and Stern, Y. (2012). Exploring the neural basis of cognitive reserve in aging. Biochim. Biophys. Acta 1822, 467-473. doi: 10.1016/j.bbadis.2011. 09.012

Steininger, S. C., Liu, X., Gietl, A., Wyss, M., Schreiner, S., Gruber, E., et al. (2014). Cortical amyloid beta in cognitively normal elderly adults is associated with decreased network efficiency within the cerebro-cerebellar system. Front. Aging Neurosci. 6:52. doi: 10.3389/fnagi.2014.00052

Su, Y., D’angelo, G. M., Vlassenko, A. G., Zhou, G., Snyder, A. Z., Marcus, D. S., et al. (2013). Quantitative analysis of PiB-PET with FreeSurfer ROIs. PLoS ONE 8:e73377. doi: 10.1371/journal.pone.0073377

Thalmann, B., Monsch, A. U., Bernasconi, F., Berres, M., Schneitter, M., ErminiFünfschilling, D., et al. (1997). CERAD - Consortium to Establish a Registry for Alzheimer's Disease Assessment Battery - Deutsche Fassung. Basel: Geriatrische Universitätsklinik.

Tombaugh, T. N. (2004). Trail Making Test A and B: normative data stratified by age and education. Arch. Clin. Neuropsychol. 19, 203-214. doi: 10.1016/S08876177(03)00039-8

Unschuld, P. G., Edden, R. A., Carass, A., Liu, X., Shanahan, M., Wang, X., et al. (2012a). Brain metabolite alterations and cognitive dysfunction in early Huntington's disease. Mov. Disord. 27, 895-902. doi: 10.1002/mds.25010

Unschuld, P. G., Joel, S. E., Liu, X., Shanahan, M., Margolis, R. L., Biglan, K. M., et al. (2012b). Impaired cortico-striatal functional connectivity in prodromal Huntington's Disease. Neurosci. Lett. 514, 204-209. doi: 10.1016/j.neulet.2012.02.095

Unschuld, P. G., Liu, X., Shanahan, M., Margolis, R. L., Bassett, S. S., Brandt, J., et al. (2013). Prefrontal executive function associated coupling relates to Huntington's disease stage. Cortex 49, 2661-2673. doi: 10.1016/j.cortex.2013.05.015

Vandenberghe, R., Van Laere, K., Ivanoiu, A., Salmon, E., Bastin, C., Triau, E., et al. (2010). 18F-flutemetamol amyloid imaging in Alzheimer disease and mild cognitive impairment: a phase 2 trial. Ann. Neurol. 68, 319-329. doi: 10.1002/ana.22068

Visser, F., Zwanenburg, J. J., Hoogduin, J. M., and Luijten, P. R. (2010). Highresolution magnetization-prepared 3D-FLAIR imaging at 7.0 Tesla. Magn. Reson. Med. 64, 194-202. doi: 10.1002/mrm.22397

Winblad, B., Palmer, K., Kivipelto, M., Jelic, V., Fratiglioni, L., Wahlund, L. O., et al. (2004). Mild cognitive impairment-beyond controversies, towards a consensus: report of the International Working Group on Mild Cognitive Impairment. J. Intern. Med. 256, 240-246. doi: 10.1111/j.1365-2796.2004.01380.x

World_Medical_Association. (1991). Declaration of Helsinki. Law Med. Health Care 19, 264-265.

Young, V. G., Halliday, G. M., and Kril, J. J. (2008). Neuropathologic correlates of white matter hyperintensities. Neurology 71, 804-811. doi: 10.1212/01.wnl.0000319691.50117.54

Zwanenburg, J. J., Hendrikse, J., Visser, F., Takahara, T., and Luijten, P. R. (2010). Fluid attenuated inversion recovery (FLAIR) MRI at 7.0 Tesla: comparison with 1.5 and 3.0 Tesla. Eur. Radiol. 20, 915-922. doi: 10.1007/s00330-009-1620-2

Conflict of Interest Statement: The authors declare that the research was conducted in the absence of any commercial or financial relationships that could be construed as a potential conflict of interest.

Received: 24 June 2014; accepted: 22 August 2014; published online: 09 September 2014.

Citation: Schreiner SJ, Liu X, Gietl AF, Wyss M, Steininger SC, Gruber E, Treyer V, Meier IB, Kälin AM, Leh SE, Buck A, Nitsch RM, Pruessmann KP, Hock C and Unschuld PG (2014) Regional Fluid-Attenuated Inversion Recovery (FLAIR) at 7 Tesla correlates with amyloid beta in hippocampus and brainstem of cognitively normal elderly subjects. Front. Aging Neurosci. 6:240. doi: 10.3389/fnagi.2014.00240

This article was submitted to the journal Frontiers in Aging Neuroscience.

Copyright (c) 2014 Schreiner, Liu, Gietl, Wyss, Steininger, Gruber, Treyer, Meier, Kälin, Leh, Buck, Nitsch, Pruessmann, Hock and Unschuld. This is an open-access article distributed under the terms of the Creative Commons Attribution License (CC BY). The use, distribution or reproduction in other forums is permitted, provided the original author(s) or licensor are credited and that the original publication in this journal is cited, in accordance with accepted academic practice. No use, distribution or reproduction is permitted which does not comply with these terms. 